\title{
Electron Transfer Dissociation Reveals Changes in the Cleavage Frequencies of Backbone Bonds Distant to Amide-to-Ester Substitutions in Polypeptides
}

\author{
Thomas A. Hansen, Hye R. Jung, Frank Kjeldsen
}

Department of Biochemistry and Molecular Biology, University of Southern Denmark, Campusvej 55, DK-5230 Odense M, Denmark

\begin{abstract}
Interrogation of electron transfer dissociation (ETD) mass spectra of peptide amide-to-ester backbone bond substituted analogues (depsipeptides) reveals substantial differences in the entire backbone cleavage frequencies. It is suggested that the point permutation of backbone bonds leads to changes in the predominant ion structures by removal/weakening of specific hydrogen bonding. ETD responds to these changes by redistributing the cleavage frequencies of the peptide backbone bonds. In comparison, no distinction between depsi-/peptide was observed using collision-activated dissociation, which is consistent with a general unfolding and elimination of structural information of these ions. These results should encourage further exploration of depsipeptides for gas-phase structural characterization.
\end{abstract}

Key words: Electron transfer dissociation, Hydrogen bonding, Depsipeptides

\section{Introduction}

$\mathrm{H}$ ydrogen bonding between main-chain amides is an ubiquitous feature of folded polypeptides (peptides/ proteins) [1]. Since polypeptide functions are mainly determined by their native structures, research directed towards deciphering the effect of hydrogen bonding has become of paramount importance in biological studies. An attractive strategy in this regard is to introduce systematic permutations of backbone amide-to-ester (A-to-E) bonds [2]. Peptides containing one or more ester backbone bonds are termed depsipeptides. The ester group in depsipeptides has significantly different chemical properties then its counter $2^{\circ}$ amide. It is a weaker stabilizer of polypeptide secondary

Electronic supplementary material The online version of this article (doi:10.1007/s13361-011-0242-5) contains supplementary material, which is available to authorized users.

Correspondence to: Frank Kjeldsen; e-mail: frankk@bmb.sdu.dk structures [3] as it cannot act as hydrogen donor as well as being a significant weaker hydrogen bond acceptor due to its lower proton affinity. Finally, its ester oxygen is partially electronegative and can therefore induce Coulomb repulsion to other amide carbonyl oxygens (Scheme 1). Since amide and ester bonds are approximately isosteric [4], it is possible to maintain the side-chain structure of depsipeptide analogues and study backbone hydrogen bonding in isolation.

Until now, depsipeptide analogues have solely been used for the characterization of the strength of individual polypeptide H-bonds in the condensed phase. However, in the solvent-free environment of the gas-phase the strength of H-bonding increases and becomes even more important for structural integrity. Gas-phase polypeptide structure characterization can be achieved with mass spectrometry coupled with ion-mobility measurements (IMS) [5], hydrogendeuterium exchange [6], blackbody infrared radiative dissociation [7], infra-red spectroscopy [8], or a variety of fragmentation techniques. In particular, techniques utilizing electron-driven fragmentation such as electron capture 


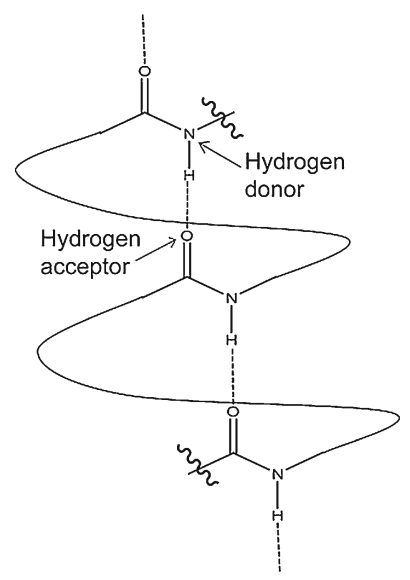

Amide-linkage

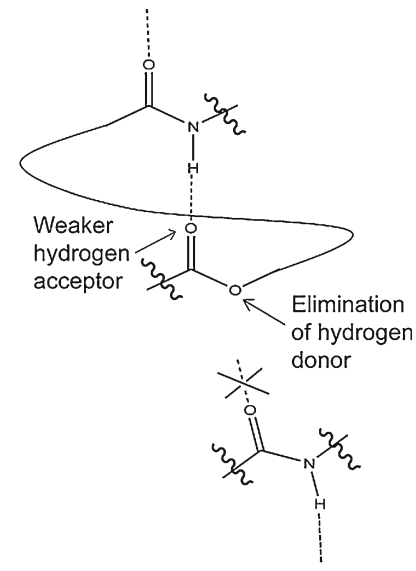

Ester-linkage
Scheme 1. Schematic representation of the perturbation of a $\mathrm{H}$-bond in a conjectural $\alpha$-helical polypeptide caused by an A-to-E backbone bond substitution

dissociation (ECD) [9] and electron transfer dissociation (ETD) [10] have become very popular. ECD and ETD involve the gas-phase reaction of poly-cations with electrons. The outcome is unstable radical intermediates that can undergo radical initiated backbone bond cleavages producing specific c-, z-type fragment ions.

ECD and ETD fragmentation techniques are attractive due to their gentle nature in cleaving the polypeptide backbone without disturbing the three-dimensional ion conformation. For instance, differences in the ECD cleavage frequency have been reported for both IMS separated conformers of protein cations [11], unfolded and re-folded ubiquitin cations [12], and for singly chiral $d$-amino acid substituted polypeptide ions [13, 14].

Based on these analytical features and the fact that polypeptide A-to-E bond substitution results in weakening/ removal of structurally involved H-bonding, we anticipate that ETD fragment ion abundances potentially can detect changes in the predominant ion conformation of depsipeptides in the gas phase. To test our prediction, we have synthesized by solid-phase synthesis eight systematically A-to-E backbone bond substituted depsipeptides and investigated in detail the cleavage frequencies of the $2+$ and $3+$ charge state ions with ETD.

\section{Experimental}

Depsi-/peptides were synthesized using a micro-scale ResPep peptide synthesizer (Intavis AG, Koeln, Germany) configured for $\mathrm{N}-\alpha$-Fmoc synthesis. A modified synthesis protocol was applied.[15] Analytes were dissolved in 50\% acetonitrile containing $0.1 \%$ formic acid and electrosprayed off-line in conc. ca. $1 \mathrm{pmol} / \mathrm{uL}$ (nanomate; Advion, Ithaca, NY). Tandem MS analysis was conducted with an Orbitrap XL ETD mass spectrometer (ThermoFisher, Bremen, Germany). ETD acquisitions were recorded in the Orbitrap with 15,000 in resolving power at $m / z 400$. For each sample, the top three most abundant ion species in the mass range $m / z$ 300-2000 were fragmented using unattended data-dependent acquisition mode. Ten acquisitions of ETD followed by supplemental activation of the ET product species (normalized collision energy $15 \%$ ) were accumulated for each peptide charge state. Ion mobility mass spectrometry was performed on a Waters Synapt G1 (Elstree, Hertfordshire, UK) instrument using a wave velocity of $300 \mathrm{~m} / \mathrm{s}$ and gas flow of $30 \mathrm{~mL} / \mathrm{min}$.

\section{Results and Discussion}

The peptide KFVGVGVGVGFK served as a good model (Table 1) as it produces complete sequence coverage in ETD from seven N-terminal $\left(\mathrm{c}^{\prime}{ }_{5}-\mathrm{c}^{\prime}{ }_{11}\right)$ and five $\mathrm{C}$ terminal $\left(\mathrm{z} \cdot{ }_{7}-\mathrm{Z} \cdot{ }_{11}\right)$ fragment ions.

Figure 1 shows as an example the summed ETD mass spectra acquired of doubly protonated KFVGVGVGVGFK and $\mathrm{V} 3 v$ in comparison. The cleavage of the ester bond is annotated with $\mathrm{z}_{10}{ }^{\# \bullet}$ according to reference [14] and displays a $37 \%$ (normalized value) higher cleavage frequency than the all-amide bonds in the same position. The enhanced cleavage frequency of the ester bond is consistent with previous results [16]. Notable is the magnitude of other amide backbone cleavages in $\mathrm{V} 3 v$. For instance, the cleavage frequency represented by $\mathrm{z}_{8}{ }_{8}$ and $\mathrm{c}_{6}{ }_{6}$ are both increased more than 3 -fold. At the same time, the backbone bond cleavage producing $\mathrm{c}^{\prime}{ }_{10}$ is reduced more than 6-fold. These observations demonstrate that the insertion of an ester bond where previously there was an amide bond can significantly influence the cleavage probability of very distant backbone bonds in ETD.

We then investigated if this finding is general among other A-to-E peptide analogous. Figure 2a displays the normalized relative cleavage frequencies in ETD of $2+$ charge state ions of the eight permutated depsipeptides. Since ETD spectra are rich with internal hydrogen atom transfers prior to fragment separation[17] we chose to sum both $\mathrm{c} \cdot / \mathrm{c}^{\prime}$ and $\mathrm{z} \cdot \mathrm{z}^{\prime}$ ion abundances. The relative cleavage frequencies were normalized to the total ion intensity of the ETD product ions. Moreover, the data in Figure 2 are obtained using identical and consecutive experimental conditions.

Table 1. Sequence of Analyzed Reference (All-Amide) Peptide and Depsipeptide Analogues

\begin{tabular}{|c|c|}
\hline Peptide/depsipeptide ${ }^{\mathrm{a}}$ & Sequence \\
\hline Reference (all amides) & $\mathrm{H}_{2} \mathrm{~N}-\mathrm{KFVGVGVGVGFK-COOH}$ \\
\hline $\mathrm{V} 3 v$ & $\mathrm{H}_{2} \mathrm{~N}-\mathrm{KF} v \mathrm{GVGVGVGFK}-\mathrm{COOH}$ \\
\hline $\mathrm{G} 4 g$ & $\mathrm{H}_{2} \mathrm{~N}-\mathrm{KFV} g$ VGVGVGFK-COOH \\
\hline $\mathrm{V} 5 \mathrm{v}$ & $\mathrm{H}_{2} \mathrm{~N}-\mathrm{KFVG} v \mathrm{GVGVGFK}-\mathrm{COOH}$ \\
\hline G6 $g$ & $\mathrm{H}_{2} \mathrm{~N}-\mathrm{KFVGVgVGVGFK-COOH}$ \\
\hline V7v & $\mathrm{H}_{2} \mathrm{~N}-\mathrm{KFVGVG} v \mathrm{GVGFK}-\mathrm{COOH}$ \\
\hline G8g & $\mathrm{H}_{2} \mathrm{~N}-\mathrm{KFVGVGVgVGFK-COOH}$ \\
\hline $\mathrm{V} 9 \mathrm{v}$ & $\mathrm{H}_{2} \mathrm{~N}-\mathrm{KFVGVGVG} v \mathrm{GFK}-\mathrm{COOH}$ \\
\hline G10g & $\mathrm{H}_{2} \mathrm{~N}-\mathrm{KFVGVGVGVgFK-COOH}$ \\
\hline
\end{tabular}

${ }^{\text {a The one-letter codes of the } \alpha \text {-hydroxy acids are lowercase italic letters and }}$ correspond to the one-letter code of the analogous $\alpha$-amino acid. 

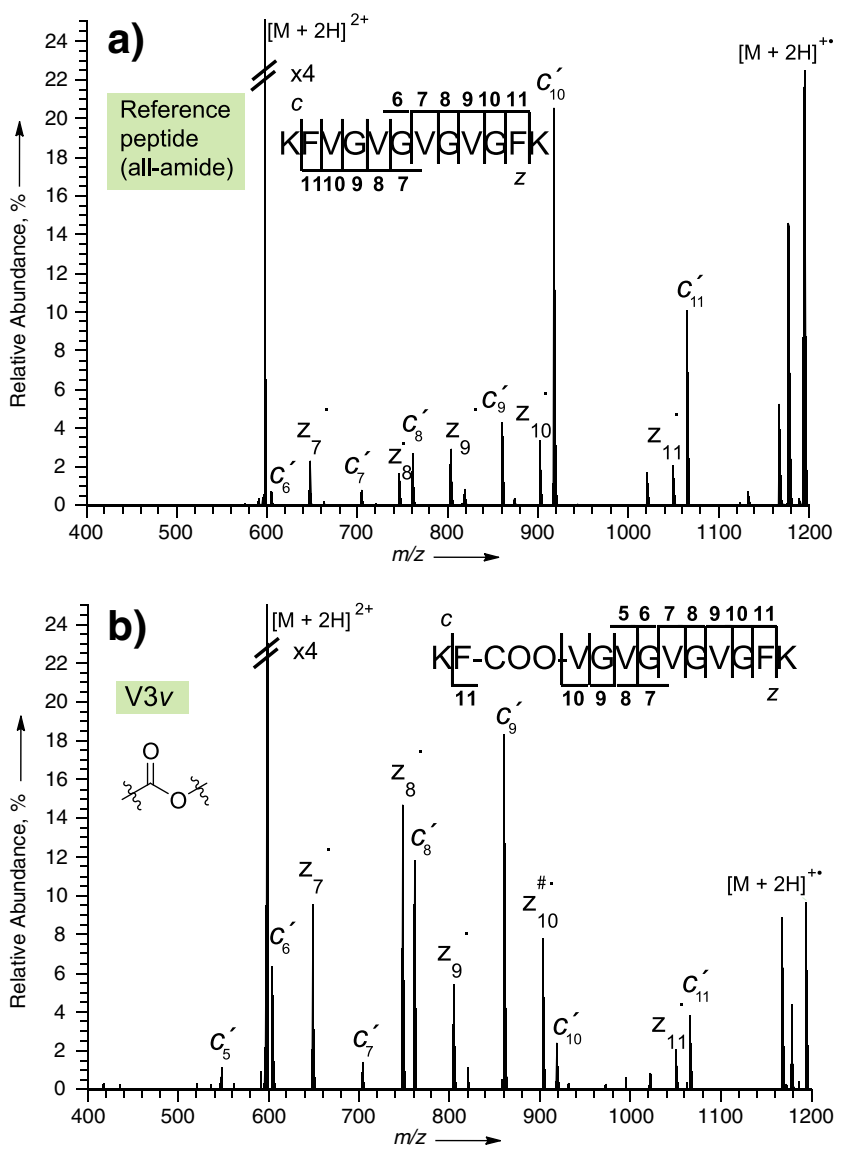

Figure 1. ETD tandem mass spectra of doubly charged (a) All-amide reference peptide KFVGVGVGVGFK and its (b) V3v depsipeptide analogue

\section{a)}

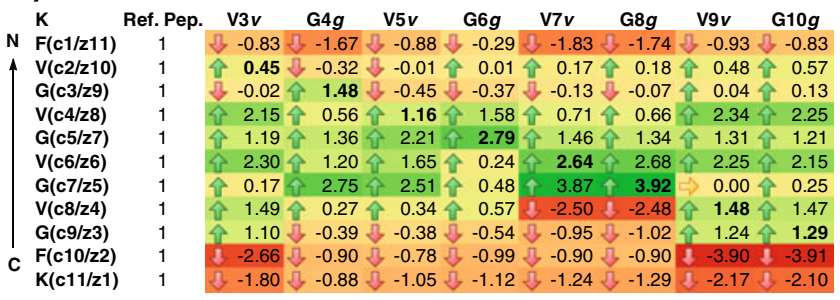

b)

\begin{tabular}{|c|c|c|c|c|c|c|c|c|c|}
\hline & Ref. Pep. & V3v & G4g & V5v & G6g & V7v & G8g & V9v & G10g \\
\hline Ref. Pep. & . $\quad 1.00$ & -0.13 & 0.63 & 0.64 & 0.38 & 0.32 & 0.31 & -0.28 & -0.26 \\
\hline V3v & & 1.00 & -0.24 & -0.07 & 0.11 & -0.49 & -0.50 & 0.98 & 0.98 \\
\hline G4 $g$ & & & 1.00 & 0.60 & 0.36 & 0.52 & 0.51 & -0.32 & -0.31 \\
\hline V5v & & & & 1.00 & 0.86 & 0.59 & 0.55 & -0.17 & -0.19 \\
\hline G6g & & & & & 1.00 & 0.17 & 0.12 & 0.06 & 0.03 \\
\hline V7v & & & & & & 1.00 & 1.00 & -0.52 & -0.53 \\
\hline G8g & & & & & & & 1.00 & -0.53 & -0.54 \\
\hline V9v & & & & & & & & 1.00 & 1.00 \\
\hline G10g & & & & & & & & & 1.00 \\
\hline
\end{tabular}

Figure 2. (a) Heat map of normalized relative differences (log2) in the cleavage frequencies of eight depsipeptides in the 2+ charge state. Values in bold present cleavages of the ester bonds. (b) Cross-correlations of cleavage frequencies of all molecular ion species
Consistent with V3v all other A-to-E analogous show significant alterations of their cleavage frequencies compared to the reference peptide. Only ETD of G6 $g$ and $\mathrm{G} 8 \mathrm{~g}$ resulted in the most abundant change arising from the ester bond cleavage. For the remaining depsipeptides, the predominant change in cleavage frequency occurred 1-8 amino acid residues away from the ester bond (average 3.5 residues). In addition, the abundance of the charge reduced species $[\mathrm{M}+2 \mathrm{H}]^{+\cdot}$ of all depsipeptides was reduced by $70 \%-85 \%$, which is an additional strong indication of lower degree of internal hydrogen bonding [12, 18, 19] in these analogues. Similarly, all depsipeptides show a 3- to 7-fold increase in the loss of $\mathrm{H}$. from $[\mathrm{M}+2 \mathrm{H}]^{+\cdot}$ compared with the reference peptide. This phenomenon has previously been suggested to correlate with the elongation of polypeptide ion conformations [20, 21].

Figure $2 b$ shows the cross-correlations of all depsi-/ peptide analogues. No strong correlation was found between the reference peptide and any of the depsipeptides $(\mathrm{r}=-0.28-0.64)$. The missing correlations support the possibility of a significant change in the H-bond architecture between the reference peptide and the depsipeptide analogues. Even if these depsi-/peptides do not have well defined secondary $\alpha$ helical or $\beta$-sheet structures it is likely that even random chain dimensions will be affected by the A-to-E substitution.

A notable observation is a surprisingly strong correlation between $\mathrm{V} 3 v, \mathrm{~V} 9 v$, and $\mathrm{G} 10 \mathrm{~g}(\mathrm{r}>0.98)$ as well as a similar correlation between $\mathrm{V} 7 v$ and $\mathrm{G} 8 g(\mathrm{r}=1)$. The indistinguishable ETD results of these analogues are puzzling. It is unclear at this point whether such results arise from a high degree of similarity of the ensemble of these ion conformations. We note that low-resolution ion-mobility mass spectrometry verified differences in the ion-molecular cross-sections of several depsi-/peptides (ranging from 264

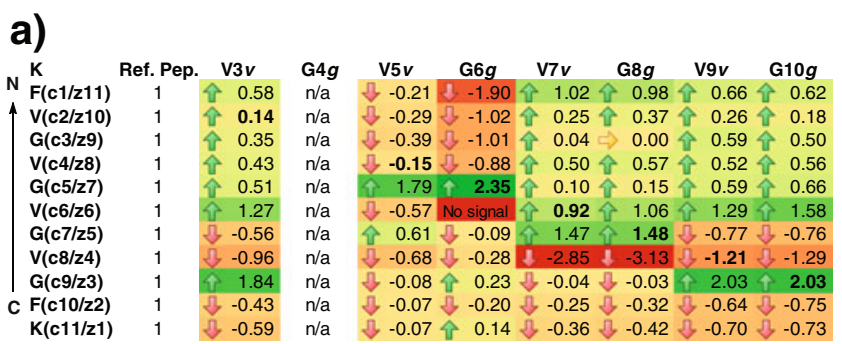

b)

$\begin{array}{llllllllll} & \text { Ref. Pep. V3v } & \text { G4 } g & \text { V5 } v & \text { G6 } g & \text { V7 } v & \text { G8 } g & \text { V9v } & \text { G10 } g \\ \text { Ref. Pep. } & 1.00 & 0.43 & \text { n/a } & 0.72 & 0.69 & 0.16 & 0.13 & 0.20 & 0.14 \\ \text { V3v } & & 1.00 & \text { n/a } & 0.22 & 0.28 & -0.03 & -0.04 & 0.97 & 0.95 \\ \text { G4g } & & & \text { n/a } & \text { n/a } & \text { n/a } & \text { n/a } & \text { n/a } & \text { n/a } & \text { n/a } \\ \text { V5 } v & & & & 1.00 & 0.88 & 0.52 & 0.50 & 0.03 & -0.01 \\ \text { G6g } & & & & & 1.00 & 0.08 & 0.05 & 0.12 & 0.08 \\ \text { V7v } & & & & & & 1.00 & 1.00 & -0.11 & -0.11 \\ \text { G8 } & & & & & & & 1.00 & -0.11 & -0.10 \\ \text { V9v } & & & & & & & & 1.00 & 0.99 \\ \text { G10 } g & & & & & & & & & 1.00\end{array}$

Figure 3. (a) Heat map of normalized relative differences (log2) in the cleavage frequencies of eight depsipeptides in the $3+$ charge state. Values in bold present cleavages of the ester bonds. (b) Cross-correlations of cleavage frequencies of all molecular ion species 
to $\left.275 \AA^{2}\right)$ although the similarity of $\mathrm{V} 9 v\left(267 \AA^{2}\right)$ and $\mathrm{G} 10 \mathrm{~g}$ $\left(275 \AA^{2}\right)$ could not be confirmed (data not shown).

A-to-E bond substitution at neighboring sites does not necessarily imply a similar ETD response. Although the correlation between $\mathrm{V} 7 v$ and $\mathrm{G} 8 g$ is 1 the correlation between G8 $g$ and V9v shows a strong anti-correlation of -0.53 . A similar amino acid context-dependence has been found in solution for other depsipeptides [2, 3, 22]. This further supports the suggestion that these ETD observations are largely structural-dependent.

The cross-correlation data of $3+$ ions (Figure 3 ) indicate a less structured ion population than those in charge state $2+$. Upon adoption of an extra proton, $3+$ ions are likely to undergo a structural elongation in order to accommodate the increased Coulomb repulsion. If so, ion conformations of depsi-/peptide analogues are expected to become more similar. This is consistent with correlations of depsipeptide analogues to the reference peptide being less deviating (lowest is 0.13 versus -0.28 in charge state $2+$ ).

In addition, the extra charge changes the fragment ion intensities although depsipeptide interrelationship does not change markedly. To demonstrate that, a poor correlation is found between the $2+$ and $3+$ reference peptides $(\mathrm{r}=0.21)$, whereas the correlation between their depsipeptide analogues in charge state $2+$ and $3+$ ranges from $r=0.55-0.88$ (Supplementary Information). The higher degree of similarity between the depsipeptide analogues fits with an already more open structure in the $2+$ charge state than the supposedly more compact reference peptide.

In conclusion, the first preliminary step towards utilization of ETD fragment ion abundances for structural characterization of a number of permutated depsipeptides in the gas phase has been demonstrated. The main results, although limited in scope, show that introduction of an A-toE bond substitution significantly affects not only the ETD cleavage frequency of the ester bond but undoubtedly also distant backbone bonds. We attribute this effect to A-to-E induced changes in the predominant ion conformer of depsipeptide analogues. No significant structural distinction between depsi-/peptide could be observed using collisionactivated dissociation $(\mathrm{r}=0.85-1.00)$ (data not shown), which is consistent with a general unfolding of polypeptide ion structures [23]. This is despite notable reduction in the ester backbone cleavage frequency in $\mathrm{CAD}$ as reported previously [16]. Finally, in order to further verify the structural predictions made by ETD, it will be necessary to include for instance extensive molecular dynamics simulations and/or high-resolution IMS of model depsipeptides. A possible contribution to the observed ETD results, which also needs further consideration, are those potentially arising from changes in the peptide electronic structure due to the A-to-E substitutions. The electronic structure of an amide $\Pi^{*}$ orbital is different to that of an ester $\Pi^{*}$ orbital. However, it is unclear at this point to what extent this difference can influence the chemical properties distant to the substitution site in peptide ions.

\section{Acknowledgments}

This work was supported by the Danish Council for Independent Research, Natural Sciences (FNU grant FK272-08-0044 to FK). Dr. James Williamson is gratefully acknowledged for careful reading of the manuscript and insightful discussion.

\section{References}

1. Dill, K.A.: Dominant forces in protein folding. Biochemistry 29, 7133-7155 (1990)

2. Deechongkit, S., Nguyen, H., Powers, E.T., Dawson, P.E., Gruebele, M., Kelly, J.W.: Context-dependent contributions of backbone hydrogen bonding to $\beta$-sheet folding energetics. Nature 430, 101-105 (2004)

3. Deechongkit, S., Dawson, P.E., Kelly, J.W.: Toward assessing the position-dependent contributions of backbone hydrogen bonding to $\beta$ sheet folding thermodynamics employing amide-to-ester perturbations. J. Am. Chem. Soc. 126, 16762-16771 (2004)

4. Ingwall, R.T., Goodman, M.: Polydepsipeptides.3. Theoretical conformational-analysis of randomly coiling and ordered depsipeptide chains. Macromolecules 7, 598-605 (1974)

5. Wyttenbach, T., von Helden, G., Bowers, M.T.: Gas-phase conformation of biological molecules: Bradykinin. J. Am. Chem. Soc. 118, 8355-8364 (1996)

6. Suckau, D., Shi, Y., Beu, S.C., Senko, M.W., Quinn, J.P., Wampler, F.M., McLafferty, F.W.: Coexisting stable conformations of gaseous protein ions. Proc. Natl. Acad. Sci. U.S.A. 90, 790-793 (1993)

7. Price, W.D., Schnier, P.D., Williams, E.R.: Tandem mass spectrometry of large biomolecule ions by blackbody infrared radiative dissociation. Anal. Chem. 68, 859-866 (1996)

8. Oh, H., Breuker, K., Sze, S.K., Ge, Y., Carpenter, B.K., McLafferty, F. W.: Secondary and tertiary structures of gaseous protein ions characterized by electron capture dissociation mass spectrometry and photofragment spectroscopy. Proc. Natl. Acad. Sci. U.S A. 99, 15863-15868 (2002)

9. Zubarev, R.A., Kelleher, N.L., McLafferty, F.W.: Electron capture dissociation of multiply charged protein cations. A nonergodic process. J. Am. Chem. Soc. 120, 3265-3266 (1998)

10. Syka, J.E.P., Coon, J.J., Schroeder, M.J., Shabanowitz, J., Hunt, D.F.: Peptide and protein sequence analysis by electron transfer dissociation mass spectrometry. Proc. Natl. Acad. Sci. U.S.A. 101, 9528-9533 (2004)

11. Robinson, E.W., Leib, R.D., Williams, E.R.: The role of conformation on electron capture dissociation of ubiquitin. J. Am. Soc. Mass Spectrom. 17, 1469-1479 (2006)

12. Breuker, K., Oh, H.B., Horn, D.M., Cerda, B.A., McLafferty, F.W.: Detailed unfolding and folding of gaseous ubiquitin ions characterized by electron capture dissociation. J. Am. Chem. Soc. 124, 6407-6420 (2002)

13. Adams, C.M., Kjeldsen, F., Zubarev, R.A., Budnik, B.A., Haselmann, K.F.: Electron capture dissociation distinguishes a single D-amino acid in a protein and probes the tertiary structure. J. Am. Soc. Mass Spectrom. 15, 1087-1098 (2004)

14. Barran, P.E., Polfer, N.C., Campopiano, D.J., Clarke, D.J., LangridgeSmith, P.R.R., Langley, R.J., Govan, J.R.W., Maxwell, A., Dorin, J.R., Millar, R.P., Bowers, M.T.: Is it biologically relevant to measure the structures of small peptides in the gas phase? Int. J. Mass Spectrom. 240, 273-284 (2005)

15. Coin, I., Beyermann, M., Bienert, M.: Solid-phase peptide synthesis: From standard procedures to the synthesis of difficult sequences. Nat. Protoc. 2, 3247-3256 (2007)

16. Kjeldsen, F., Zubarev, R.A.: Effects of peptide backbone amide-to-ester bond substitution on the cleavage frequency in electron capture dissociation and collision-activated dissociation. J. Am. Soc. Mass Spectrom. (2011). doi:10.1007/s13361-011-0151-7

17. Swaney, D.L., McAlister, G.C., Wirtala, M., Schwartz, J.C., Syka, J.E. P., Coon, J.J.: Supplemental activation method for high-efficiency electron-transfer dissociation of doubly protonated peptide precursors. Anal. Chem. 79, 477-485 (2007) 
18. Skurski, P., Sobczyk, M., Jakowski, J., Simons, J.: Possible mechanisms for protecting $\mathrm{N}-\mathrm{C}-\rightarrow$ bonds in helical peptides from electron-capture (or transfer) dissociation. Int. J. Mass Spectrom. 265, 197-212 (2007)

19. Horn, D.M., Breuker, K., Frank, A.J., McLafferty, F.W.: Kinetic intermediates in the folding of gaseous protein ions characterized by electron capture dissociation mass spectrometry. J. Am. Chem. Soc. 123, 9792-9799 (2001)

20. Breuker, K., Oh, H.B., Cerda, B.A., Horn, D.M., McLafferty, F.W.: Hydrogen atom loss in electron-capture dissociation: A fourier transformion cyclotron resonance study with single isotopomeric ubiquitin ions. Eur. J. Mass Spectrom. 8, 177-180 (2002)
21. Mihalca, R., Kleinnijenhuis, A.J., McDonnell, L.A., Heck, A.J.R., Heeren, R.M.A.: Electron capture dissociation at low temperatures reveals selective dissociations. J. Am. Soc. Mass Spectrom. 15, 1869-1873 (2004)

22. Scheike, J.A., Baldauf, C., Spengler, J., Albericio, F., Pisabarro, M.T., Koksch, B.: Amide-to-ester substitution in coiled coils: The effect of removing hydrogen bonds on protein structure. Ang. Chem. Int. Ed. 46, 7766-7769 (2007)

23. Burlet, O., Yang, C.Y., Gaskell, S.J.: Influence of cysteine to cysteic acid oxidation on the collision-activated decomposition of protonated peptides-evidence for intraionic interactions. J. Am. Soc. Mass Spectrom. 3, 337-344 (1992) 